CIUDAD Y TERRITORIO

ESTUDIOS TERRITORIALES

ISSN(P): 1133-4762; ISSN(E): 2659-3254

Vol. LII, № 203, primavera 2020

Págs. 71-78

https://doi.org/10.37230/CyTET.2020.203.06

CC BY-NC 4.0

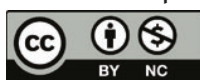

\title{
Género y urbanismo en la experiencia valenciana
}

\section{Carmen Antequera-Verdeguer}

Directora de la Mesa de Género de la Conselleria de Vivienda, Obras Públicas y Vertebración del Territorio. Generalitat Valenciana

RESUMEN: Este artículo describe la experiencia de integración de la perspectiva de género en el urbanismo en la Comunidad Autónoma Valenciana. Este proceso se inició en 2015 desde la Conselleria de Vivienda, Obras Públicas y Vertebración del Territorio (CVOPVT), en cuyo seno se constituyó, desde el mismo inicio de legislatura y por iniciativa de la propia Consellera, $M^{\mathrm{a}}$ José Salvador, una Mesa de Género de alto nivel. Esta Mesa tiene el mandato de trabajar las competencias del departamento con perspectiva de género, en colaboración con la unidad de igualdad de la CVOPVT. Es un grupo multidisciplinario que persigue una vía de aproximación a las competencias de la Conselleria desde la experiencia de las mujeres, que históricamente han tenido escasa participación en el diseño de la ciudad y el territorio. Con la creación de la Mesa, se decidió explorar y abordar al máximo nivel la perspectiva de género en la vivienda, la ciudad, el territorio y la movilidad. En este artículo se describe el proceso de puesta en marcha y aplicación de estas políticas.

PALABRAS CLAVE: Perspectiva de género. Urbanismo. Vivienda. Gobierno regional. Movilidad.

\section{Gender and urban planning in the Valencian experience}

ABSTRACT: This article describes the experience of integrating the gender perspective in urban planning in the Valencian Autonomous Community. This process was begun in 2015 by the Department of Housing, Public Works and Vertebration of the Territory (CVOPVT), where a high level Gender Bureau was constituted, from the very beginning of the legislature and at the initiative of the Consellera $M^{a}$ J José Salvador. This Bureau has the mandate to work the competences of the department with a gender perspective, in collaboration with the equality unit of the CVOPVT. It is a multidisciplinary group that pursues an approach to the competences of the Conselleria from the experience of women, who historically have had little participation in the design of the city and territory. With the creation of the Bureau, it was decided to explore and address -at the highest level- the gender perspective in housing, the city, the territory and mobility. This article describes the process of implementation and application of these policies.

KEYWORDS: Gender mainstreaming. Urban planning. Housing. Regional government. Mobility.

Recibido: 04/09/.2019; Revisado: 10.12.2019

Correo electrónico: antequera.verdeguer@gmail.com NºRCID: https://orcid.org/0000-0002-2064-460X 


\section{Creación de las estructuras: la Mesa de Género de la Conselleria de Vivienda, Obras Públicas y Vertebración del Territorio (CVOPVT) y las unidades de igualdad}

a Mesa de Género de la CVOPVT está formada por un grupo de mujeres que ocupan cargos de responsabilidad en los sectores relevantes con poder de decisión en el diseño y planificación de la ciudad y el territorio de la administración regional valenciana. El grupo que integra entidades con capacidad de ejecución y operativa considerables, que combina puestos políticos, conocimientos técnicos, dotaciones y recursos. ${ }^{1}$

Los principales objetivos de la Mesa son cuatro: 1) Sensibilizar a los diferentes sectores involucrados en la gestión urbana: administración, técnicos, ciudadanos. 2) Diagnosticar el estado de la cuestión e identificar nichos de colaboración. 3) Implementar reformas legislativas coordinadas. 4) Incentivar acciones en materia de diseño de espacios urbanos.

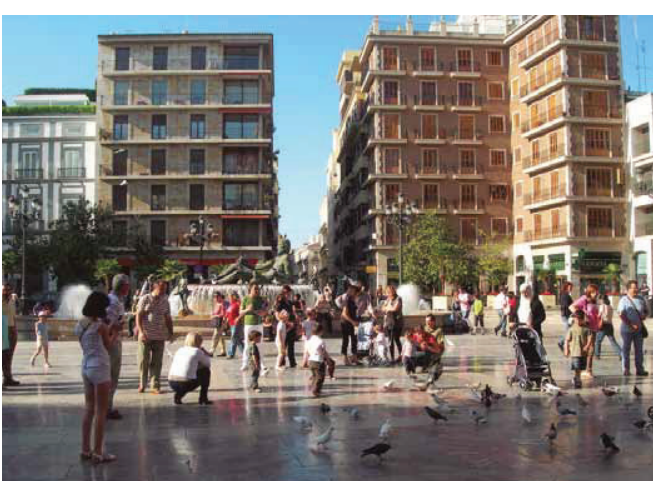

FIG. 1/ Foto Subdirección General. Políticas Urbanas.

Fuente: MINISTERIO DE TRANSPORTES, MOVILIDAD y Agenda URBANA.

\footnotetext{
${ }^{1}$ Las componentes de la Mesa de Género a lo largo de su trayectoria (2015-2019) han sido: Maria José Salvador Rubert, Consellera de Vivienda, Obras Públicas y Vertebración del Territorio; Carmen Antequera Gómez, Asesora de la Consellera, directora-coordinadora de la Mesa; Rebeca Torró Soler, Directora General de Vivienda, Rehabilitación y Regeneración Urbana; Blanca Marín Ferreiro, desde Agosto 2015 a Septiembre 2016, Directora General de la Entidad de Infraestructuras de la Generalitat Valenciana; Begoña Serrano Lanzarote, Directora del Instituto Valenciano de la Edificación Helena Beunza Ibáñez, desde Septiembre 2016 hasta Julio 2018, Directora de la Entidad Valenciana de Vivienda y Suelo; Rosa Pardo, Directora General de Urbanismo desde Julio de 2018; Laura Soto Francés, desde Julio 2018, Directora de la Entidad Valenciana de Vivienda y Suelo; Eva Álvarez,
}

Tomando como referencia las líneas estratégicas que inspiran la Nueva Agenda Urbana, la ciudad inclusiva, la ciudad innovadora y la ciudad sostenible, la Mesa inició su actividad impulsando diferentes acciones divulgativas y legislativas: jornadas, exposiciones, publicaciones, guías, modificaciones de regulación, premios para las actuaciones urbanas con perspectiva de género, concursos públicos para habilitar vivienda con perspectiva de género, entre otras.

En paralelo a la creación de la Mesa de la CVOPVT, se decidió la creación de las Unidades de Igualdad, estipuladas en la Ley orgánica 3/2007, de 22 de marzo, para la igualdad efectiva de mujeres y hombres, que no habían sido creadas por los anteriores ejecutivos. Con fecha de 20 de octubre de 2015 se constituyeron dichas unidades en todas las Consellerias, adscritas a la Subsecretaría correspondiente, a través del Gabinete Técnico. Este Gabinete Técnico es el engranaje entre la dirección política de las Consellerías y la estructura técnica. Al tener carácter horizontal, facilita la transversalidad.

Así se da cumplimiento al objetivo fijado en la legislación estatal, y también en la autonómica, la Ley 9/2003, de 2 de abril, de la Generalitat Valenciana, para la Igualdad entre Mujeres y Hombres, que establece:
"regular y hacer efectivo el principio de igualdad de mujeres y hombres en la Comunitat Valenciana, es- tablecer los principios generales que deben orien- tar dicha igualdad, determinar las acciones básicas que deben ser implementadas, así como estable- cer la organización administrativa de la igualdad de oportunidades entre mujeres y hombres en la Comunitat Valenciana".

Entre las competencias de las Unidades de Igualdad se encuentran, en primer lugar, la promoción

Arquitecta, Profesora del departamento de Proyectos Arquitectónicos de la Universitat Politècnica de València; Marina Calatayud Cuesta, responsable de la Unidad de Igualdad; María Vicenta Gil Vila, Jefa de Servicio de Planificación Económica y Regeneración Urbana; Pilar Álvarez Montero, Subdirectora de Puertos, Aeropuertos y Costas; Ana Puertas Medina, Técnica del Servicio de Infraestructura Verde y Paisaje; Sira Azara Escrivá, Técnica del Servicio de Planificación Económica y Regeneración Urbana; Nati Noverges Doménech, Unidad de Igualdad; María Verchili Martí, Unidad de Igualdad; Pepa Bertolin Corbatón, Unidad de Igualdad; Carolina Mateo Cecilia, Técnica del Instituto Valenciano de la Edificación; Maria Flores Fillol, Técnica de la Entidad Valenciana de Vivienda y Suelo; Mar Alonso Monterde, Técnica del Instituto Valenciano de la Edificación. 
de la elaboración de estudios de investigación e informes técnicos de diagnóstico de la situación de las mujeres, en relación con las áreas de actividad de cada departamento, con la finalidad de detectar y corregir posibles situaciones de desigualdad entre mujeres y hombres. En segundo lugar, la supervisión y propuesta de medidas para la incorporación de la perspectiva de género en los contratos, subvenciones, convenios, acuerdos, gestión de recursos humanos, asesoramiento en materia de lenguaje inclusivo, supervisión en la elaboración de informes sobre impacto por razón de género en las Leyes y disposiciones de carácter general y análisis del impacto de los resultados que su realización ha tenido con respecto al género.

Desde el inicio de la legislatura en 2015 el Gobierno Valenciano entrante toma la decisión de transversalizar las políticas para la igualdad efectiva de hombres y mujeres en toda su acción política. Así, la Ley 13/2016, de 29 de diciembre, de medidas fiscales, de gestión administrativa y financiera, y de organización de la Generalitat, llamada "Ley de Acompañamiento" introduce la voluntad de aplicar la igualdad efectiva entre hombres y mujeres a todos los ámbitos políticos y actos administrativos. En su Capítulo XII modifica la ley para la Igualdad, de la C.V. estableciendo:

"Los proyectos normativos incorporarán un informe de impacto por razón de género que se elaborará por el departamento o centro directivo que propone el anteproyecto de ley o proyecto de norma, plan o programa de acuerdo con las instrucciones y directrices que dice el órgano competente en la materia, y deberá acompañar la propuesta desde el inicio del proceso de tramitación".

Para facilitar el cumplimiento de esta obligación, la Subsecretaría de la Conselleria de Vivienda, Obras Públicas y Vertebración del Territorio, a través de la Unidad de Igualdad, elaboró y difundió entre los órganos directivos los procedimientos para el ejercicio de las funciones atribuidas reglamentariamente a la Unidad de Igualdad en materia de Informes de Impacto de Género y fomento del lenguaje no sexista, así como la plantilla para guiar la elaboración de los informes y las instrucciones para fomentar y asegurar el uso de lenguaje no sexista y no discriminatorio en los documentos e informes elaborados por la propia Conselleria.

La Unidad de Igualdad es determinante para poder llevar a cabo los objetivos marcados para la legislatura. Su implicación ha ido más allá de las tareas propias encomendadas, ya que ha funcionado como verdadero dinamizador y promotor de la implantación de la perspectiva de género en el departamento. Bajo su impulso, los informes de impacto de género de las leyes, de los Planes de Movilidad, de los Presupuestos, contratos, subvenciones, acuerdos, gestión de recursos humanos, etc., han dejado de ser un trámite más administrativo.

Todas las normas y planes elaborados durante esta legislatura han contado con los correspondientes informes, asesorados por la Unidad de Igualdad, que ha facilitado, siguiendo el procedimiento acordado, la información para el análisis de la situación de partida en la materia objeto de la norma o plan, ha orientado el enfoque, ha realizado sugerencias sobre contenidos, etc, convirtiendo los informes en auténticos documentos con contenido sustantivo que no se limitan a cumplir un trámite meramente formal.

La Unidad de Igualdad ha asesorado entre otros los informes de impacto de género de la Ley de Seguridad Ferroviaria, Ley de la Huerta de València, Ley del taxi, Ley por la función social de la vivienda, modificación de la Ley de Ordenación del Territorio, Urbanismo y Paisaje, Plan de Acción Territorial de Ordenación y Dinamización de la Huerta de Valencia, UNEIX: Programa estratégico para la mejora de la Movilidad, Infraestructuras y Transporte. (ver nota 5).

La Unidad ha trabajado igualmente en el fomento del uso del lenguaje no sexista y no discriminatorio con la revisión de todo tipo de texto normativo, los planes de movilidad metropolitanos, con sus encuestas asociadas, entre otros. También ha hecho seguimiento y evaluación del grado de cumplimiento de la normativa vigente en materia de igualdad entre hombres y mujeres. Ha evaluado el Plan de Igualdad de la Generalitat Valenciana. También se ha actuado respecto de la información estadística, instando a las unidades administrativas a que gestionen la información susceptible de ser desagregada por sexos para los datos estadísticos y cumplir con este aspecto con la Ley Orgánica 2/2007 de 22 de marzo, para la igualdad efectiva de mujeres y hombres.

\section{Innovar, formar, participar}

Como resultado de las deliberaciones de la Mesa de Género se decidió poner en marcha medidas para fomentar la innovación en el diseño de la vivienda. Para ello se ha organizado un "Concurso de Nuevas estrategias habitacionales para estu- 
diantes de arquitectura"2, cuyo objetivo es explorar ideas innovadoras dirigidas a diseñar y adaptar viviendas del Parque Público de la Generalitat, desde la perspectiva de género, como proyecto piloto. El objetivo es llevar a la práctica desde la administración temas abordados desde el punto de vista teórico, creando un laboratorio de ideas innovadoras que permitan adaptar la normativa actual del diseño de viviendas a unos parámetros que tengan en la diversidad y la perspectiva de género su inspiración principal.

Este concurso ha sido un aliciente para pensar sobre la vivienda con un enfoque de género, considerando que las tipologías de vivienda existentes responden la mayoría de las veces a estructuras familiares tradicionales de cuatro o cinco miembros, que no se ajustan a muchas realidades actuales. Además, cada vez hay más personas mayores que viven solas en viviendas no adaptadas a sus necesidades y que les impiden vivir con autonomía durante más tiempo.

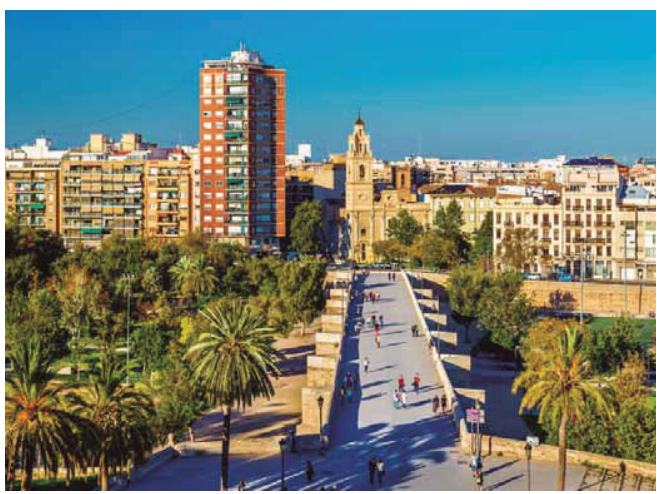

Fig. 2/ Foto Subdirección General. Políticas Urbanas.

Fuente: MinISTERIO DE TRANSPORTES, MoviLIDAD Y AgENDA URBANA.

De ahí la necesidad de incentivar a los futuros arquitectos y arquitectas a la investigación. A través de la Mesa de Género se impulsó este concurso dirigido a las escuelas de arquitectura de la Comunitat Valenciana, centrándose en dos aspectos: la rehabilitación de edificios y la profundización en el diseño de viviendas, con enfoques integradores que ofrecieran soluciones innovadoras para atender a la diversidad familiar actual.

\footnotetext{
${ }^{2}$ Concurso de Nuevas estrategias habitacionales para estudiantes de arquitectura CaLAB.

URL: https://www.five.es/concurso-neh-propuestas-seleccionadas/ [fecha de consulta: 28-06-2019]

${ }^{3}$ Concurso de regeneración Urbana en el entorno de la plaza Ciudad de Brujas, Lonja de la Seda, Mercado Central e Iglesia de los Santos Juanes
}

Con el "Concurso de regeneración Urbana en el entorno de la plaza Ciudad de Brujas, Lonja de la Seda, Mercado Central e Iglesia de los Santos Juanes"3 también hemos podido trabajar en una fórmula pionera que hasta la fecha no se había ensayado en la administración autonómica. Se trata de un proceso dirigido a acometer una operación de regeneración urbana en el entorno de la Plaza de Brujas que pretende acabar con una situación de degradación de un conjunto arquitectónico de incalculable valor en el centro de Valencia, en una zona con varios Bienes de Interés Cultural, con un edificio que es Patrimonio de la Humanidad como es la Lonja de la Seda, con el Mercado Central de estilo modernista y la Iglesia de San Juan del Mercado, declarada Monumento Artístico Nacional.

La fórmula del concurso en dos fases ha sido un proceso elaborado que ha permitido integrar la participación pública y también dar oportunidad al talento. El resultado de esta importante operación de regeneración urbana contribuirá a mejorar la calidad de vida de la ciudad con un entorno más amable con la ciudadanía que tendrá en cuenta las necesidades de género, la movilidad sostenible y los espacios verdes.

El proyecto de regeneración urbana de este entorno en la milla de oro arquitectónica de la ciudad de Valencia, que ya ha sido entregado al Ayuntamiento para su ejecución, ha nacido con la máxima participación ciudadana y vecinal. En este proceso todas las personas interesadas han podido participar y han conocido la evolución del concurso, aportando sugerencias, expresando sus opiniones, que han sido tenidas en cuenta por los redactores de los proyectos. Cada equipo que participó en el concurso tuvo un mes para reelaborar su documento y entregar un anteproyecto. Se dirimió con intervención de jurado, para la selección de la propuesta y posterior redacción del proyecto y dirección de las obras. Los criterios de valoración se estipularon de la siguiente manera: hasta 15 puntos para objetivos generales; hasta 25 puntos Espacio público y paisaje urbano; hasta 20 puntos en Movilidad, conexión y articulaciones; hasta 20 puntos en Sostenibilidad y criterios ambientales; hasta 10 puntos en Usos y actividades; y hasta 10 puntos en Integración y participación social. 
Los tres proyectos preseleccionados estuvieron expuestos en la Lonja donde los propios autores mediante un servicio de audioguía explicaban las diferentes propuestas. Además, se habilitó una urna y una dirección de correo para recoger las sugerencias de los ciudadanos. Después de este proceso el jurado se encargó de seleccionar la propuesta ganadora que en este caso fue para el proyecto "Confluencias", de las arquitectas Elisabet Quintana y Blanca Peñín, que tiene el objetivo de dar un carácter unitario a todo el entorno mediante elementos como la recuperación de la escalera de la antigua plaza de acceso a San Juan del Mercado y la adecuación de "les covetes" para convertirlas en espacios de interpretación. El proyecto también activará el espacio público de la avenida del Oeste para adecuarlo al espacio residencial del barrio con pavimentos peatonales y vegetales. La plaza de Brujas estará formada por un elemento pergolado que recoja todos los elementos en superficie como el aparcamiento, la parada de autobús y otros elementos del entorno.

\section{Herramientas y difusión: el "SET" para incorporar la perspectiva de género y los "Premios Vivienda, Movilidad y Urbanismo con perspectiva de género"}

El "Set para introducir la perspectiva de género en el proceso urbano"4, es una de los primeras herramientas que elaboró la Mesa de Género y presentado con motivo del 8 de marzo de 2016. Es un documento a modo de guía, accesible en la web de la Generalitat y que recoge en siete capítulos de forma descriptiva y con ejemplos cómo abordar el género en el hecho urbano. Modelo de ciudad, Percepción de Seguridad, Espacios Públicos y Accesibilidad, Movilidad, Vivienda, Representación y señalización urbana y Participación ciudadana, son los siete ejes sobre los que se articula este documento. Sus destinatarios son los técnicos involucrados en los procesos de creación de nuevos ámbitos urbanos o de regeneración, bien sean equipos de ayuntamientos, profesionales de la arquitectura, el urbanismo o la promoción inmobiliaria.

Para dar respuesta a una demanda de información detectada proveniente de la administración local a la hora de abordar el enfoque de género en la ciudad, se planteó un documento práctico que compendiara información básica mientras se legislaba la materia en la Ley de Ordenación del Territorio. El SET es una síntesis esquemática que recopila análisis, propuestas, bibliografía, materiales de jornadas, seminarios. Con el SET ofrecemos un marco de soporte para mejorar la capacitación técnica, la formación y concienciación en la perspectiva de género aplicada al urbanismo, arquitectura y movilidad. Las medidas propuestas tienen un efecto demostrativo, didáctico y ejemplar que hace visible diversas posibilidades de aplicación en todas las fases del proceso urbanístico, desde el diseño, la participación, la planificación y ejecución, hasta la evaluación y seguimiento.

Por otro lado, la creación de los "Premios Vivienda, Movilidad y Urbanismo con perspectiva de Género" es otra de las acciones impulsadas con el objetivo de difundir e incentivar a los ayuntamientos, colectivos, asociaciones, universidades, tercer sector, medios de comunicación, para pensar conjuntamente cómo incorporar de forma igualitaria el diseño de los espacios públicos. Una manera más de hacer visible y concienciar medidas encaminadas a promover el género en la transformación urbana y en la mejora física y social de los entornos, los barrios, pueblos y ciudades.

Se premian planes de movilidad, señalética igualitaria, equipamientos y mobiliario urbano, diseño igualitario de parques infantiles o patios de colegios, diseño igualitario de parques saludables para personas mayores, normativas y procedimientos administrativos que incluyan la perspectiva de género; concursos de ideas para regeneración urbana con perspectiva de género; iniciativas tendentes a la equidad en nomenclátores de vías públicas; guías o recomendaciones; actividades reivindicativas y de empoderamiento de los espacios públicos, procesos participativos en la toma de decisiones en el diseño de las intervenciones, entre otras.

\section{Medidas legislativas}

Legislar con perspectiva de género ha sido una de las prioridades durante la legislatura 2015-2019, con especial atención a las mujeres más vulne${ }^{4}$ SET para introducir la perspectiva de género en el proceso
urbano [Documentos de trabajo]. Valencia: Conselleria de Vivienda, Obras Públicas y Vertebración del Territorio, 2017. 
rables $^{4}$. La Ley 2/2017 de la Función social de la vivienda, incluye condiciones para garantizar los derechos a la vivienda a las víctimas de violencia de género y se reconoce este derecho a disfrutar de una vivienda asequible, digna y adecuada (art. 2). También se reconocen una serie de medidas para garantizar la protección en procesos de ejecución hipotecaria o desahucios a mujeres víctimas de violencia de género (art, 12), y también se han adecuado las resoluciones de ayudas al alquiler que oscilan en torno al $30 \%$ del total. También se ha facilitado el acceso prioritario a las viviendas del parque público de la Generalitat.

Ley $13 / 2017$, de 8 de noviembre, del Taxi de la Comunitat Valenciana, regula este servicio público, que es a su vez es un sector económico tradicionalmente masculinizado, instando a la administración a adoptar medidas de acción positiva para las mujeres en el acceso a la licencia de actividad del taxi, (art. 7) garantizando así nuevos referentes y equilibrio en la esfera pública

La Ley de Ordenación del Territorio, Ley 1/2019, de 5 de febrero, de la Generalitat, de modificación de la Ley $5 / 2014$, de 25 de julio, de ordenación del territorio, urbanismo y paisaje de la Comunitat Valenciana, ha sido la oportunidad para introducir la perspectiva de género para avanzar en la planificación. El texto consagra en diferentes artículos la perspectiva de género como eje fundamental a la hora de dar una nueva mirada introduciendo criterios y reglas para la planificación de las actuaciones de rehabilitación, regeneración y renovación urbana con perspectiva de género.

El urbanismo para las personas; la red de espacios comunes; la proximidad de las actividades de la vida cotidiana; la combinación de usos y actividades; la seguridad en el espacio público; la habitabilidad del espacio público; los equipamientos; la vivienda y la movilidad, están recogidos en un Anexo en la Ley para la implantación transversal de la perspectiva de género en la práctica urbanística de manera multiescalar, interdisciplinaria y participativa. Esta incorporación, como uno de los grandes objetivos de la ley, atiende a los

\footnotetext{
${ }^{5}$ Principal legislación y planificación elaborada y aprobada durante la legislatura 2015-2019:

- Ley $7 / 2018$, de 26 de marzo, de la Generalitat, de seguridad ferroviaria:

http://www.dogv.gva.es/va/disposicio-consolidada?signatura=003 134/2018\&idioma=es\&L=1\&url lista=

- Ley 5/2018, de 6 de marzo, de la Generalitat, de la Huerta de València: $h$ ttp://www.dogv.gva.es/datos/2018/03/12/pdf/2018 2459.pdf

- Ley 13/2017, de 8 de noviembre, de la Generalitat, del Taxi de la - Ley $13 / 2017$, de 8 de novit
Comunitat Valenciana:

http://www.dogv.gva.es/es/disposicio-consolidada?signatura=009 695/2017\&idioma=es\&L=1\&url lista=

- Ley 2/2017, de 3 de febrero, de la Generalitat, por la función social de la vivienda de la Comunitat Valenciana:
}

principios de igualdad y reconoce los papeles en los diferentes usos de las ciudades y del territorio con enfoques integrados, sostenibles medioambiental y económicamente para centrarse en las personas teniendo en cuenta su género y edad.

En la Ley 5/2018, de 6 de marzo, de la Generalitat, de la Huerta de València, una de las leyes de ordenación del territorio más esperadas y que por fin protege un paisaje productivo y cultural de incalculable valor y que estaba seriamente amenazado por la especulación urbanística junto al abandono de la actividad agraria. También se ha trabajado el género en este paisaje único. Un espacio vivo en el que las mujeres han desarrollado una actividad productiva y económica, invisibilizada a través de los siglos, cuyo ejemplo más palmario es la ausencia de mujeres en el Tribunal de las Aguas, órgano consuetudinario de resolución de conflictos de riego. En el art. 5.2 la ley otorga a los poderes públicos el deber de fomentar las acciones tendentes a la mejora de las condiciones económicas, sociales y culturales del agricultor y agricultora. La creación del Ente Gestor de l'Horta supone una oportunidad para promover la representación paritaria y el fomento de la presencia de la mujer en la toma de decisiones.

\section{Visibilizar y nombrar}

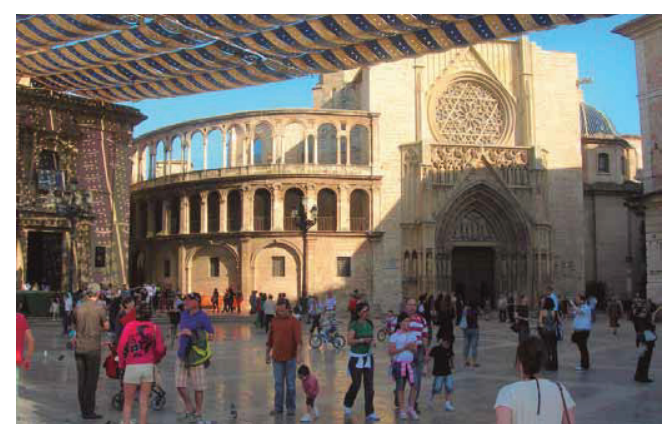

FIG. 3/ Foto Subdirección General. Políticas Urbanas.

Fuente: Ministerio de Transportes, MoviLIDAD y Agenda URBAnA.

http://www.dogv.gva.es/es/disposicio-consolidada?signatur a=001392/2017\&idioma=es\&L=1\&url lista $=$

Ley $1 / 2019$, de 5 de febrero, de la Generalitat, de modificación de la Ley 5/2014, de 25 de julio, de ordenación del territorio, urbanismo y paisaje de la Comunitat Valenciana:

http://www.dogv.gva.es/es/disposicio-consolidada?signatura=00 6922/2014\&idioma=es\&L=1\&url lista=

- Plan de Acción Territorial de Ordenación y Dinamización de la Huerta de Valencia:

http://www.dogv.gva.es/datos/2018/12/20/pdf/2018 11893.pdf

- UNEIX: Programa estratégico para la mejora de la Movilidad, Infraestructuras y Transporte:

http://www.habitatge.gva.es/es/uneix

[fecha de consulta: 28-06-2019]. 
Por último, desde la Mesa de género se ha impulsado un proceso de cambio de denominación de los grupos de vivienda pertenecientes al Parque Público. Algo tan relevante en la memoria urbana como es la designación de los Grupos de viviendas del parque público de la Comunitat Valenciana estaba copado por hombres que se habían significado por su pertenencia a la dictadura. La mayoría de grupos de viviendas, pese a haber sido transferidos en 1984, mantenían el nomenclátor de la dictadura franquista, a pesar de tener las competencias transferidas sobre patrimonio arquitectónico, control de calidad de edificación y vivienda y de cuantas normas se habían ido incorporando en materia de gestión y administración del parque público de viviendas de la Comunitat Valenciana. Con todo, ninguna norma sin embargo había previsto el cambio de denominación de los grupos de vivienda de promoción pública cuya titularidad se había transferido por el Estado. Es decir, los grupos de viviendas se continuaban llamando, por ejemplo, Batalla Brunete, Antonio Rueda, Salas Pombo, General Yagüe, Alcázar de Toledo, Primo de Rivera.

La situación encontrada ofrecía una oportunidad para trabajar conjuntamente la memoria democrática y la igualdad entre hombres y mujeres en lo referido a modificar los patrones socioculturales y visibilizar a la mujer. La Ley Orgánica 3/2007 para la igualdad efectiva entre hombres y mujeres que en sus artículos 24 y 31 hace referencia a la acción administrativa en las políticas educativas, urbanas, de ordenación territorial y vivienda, establece el deber por parte de las administraciones públicas de tener en cuenta la perspectiva de género en el ámbito de la ciudad, así como el reconocimiento del papel de las mujeres en la historia. Esta disposición, junto con lo establecido por la Ley 14/2017 de 10 de noviembre de Memoria democrática y para la convivencia de la Comunitat Valenciana, viene a fundamentar y resolver la cuestión.

En el nomenclátor del Parque Público de viviendas se ha realizado mediante sendas resoluciones el cambio de denominación de los grupos de vivienda de promoción pública ${ }^{6}$, así como la retirada o eliminación de los correspondientes elementos colocados en ellos. La renovación de los nombres de 54 grupos de viviendas se ha hecho en dos fases, dando visibilidad a mujeres destacadas en la lucha por la democracia y la igualdad. Mujeres que tuvieron, en contra de los signos de su tiempo, un papel determinante en el ámbito artístico, políti-

${ }^{6}$ Nuevo Nomenclátor Parque Público de Viviendas [Libros]. València: Conselleria d'Habitatge, Obres Públiques i Vertebració del Territori: Entitat Valenciana d'Habitatge i Sòl, 2019 co, empresarial o cultural. Los nombres incluyen no solamente a mujeres ilustres como Matilde Salvador, Ascensión Chirivella, Manuela Solis, Asunción Cruañes o Jane Jacobs, entre otras, sino también a mujeres anónimas, que desempeñaron de manera colectiva un papel protagonista con frecuencia silenciado, reconociendo también la labor de las trabajadoras del mar, el campo, el textil, la seda o las palmas, por ejemplo, o de las maestras Republicanas, las mujeres Sindicalistas, las Constituyentes, las Sufragistas, las Modernistas y también conceptos como Sororidad o fechas significativas en la lucha feminista como el 8 de marzo o el 25 de noviembre.

\section{Conclusión}

Este artículo ha descrito brevemente las principales iniciativas promovidas en la Conselleria de Vivienda Obras Publicas y Vertebración del Territorio a través de la Mesa de Género, en ámbitos que incluyen la vivienda, la vertebración del territorio o la movilidad. El fin último es generar un cambio de paradigma en la gestión pública situando el género como poderoso motor de cambio que nos lleva a interrogarnos sobre cómo nos afectan los ámbitos de nuestra convivencia.

Este trabajo ha sido llevado a cabo por un grupo de mujeres que desde sus distintos puestos de responsabilidad en la administración de la Generalitat han trabajado en equipo, de manera comprometida con la innovar e impulsar estas buenas prácticas: Maria José Salvador, Blanca Marín, Begoña Serrano, Laura Soto, Helena Beunza, Rebeca Torró, Rosa Pardo, Marina Calatayud, Carolina Mateo, Maria Flores, Marvi Gil, Eva Álvarez, Pilar Álvarez, MarAlonso, Ana Puertas, SiraAzara, Nati Noverges, María Verchili y Pepa Bertolin.

\section{Bibliografía}

Ayuntamiento de Fuenlabrada (2007): Viviendas que concilian. La perspectiva de género aplicada a las viviendas de nueva construcción.

http://www.redmujer.org.ar/pdf publicaciones/art 44.pdf

Col-lectiu Punt 6: Dones treballant. Guía de reconeiximent urbà amb perspectiva de gèner. https://punt6.files.wordpress.com/2011/03/donestreballant-final-baixa.pdf

EMAKUNDE: Seminario Urbanismo inclusivo: Las calles tienen género.

URL: http://www.evha.es/portal/castellano/ficheros/nomenclator/ nomenclator-ppv.pdf

[fecha de consulta: 28-06-2019] 


\section{Estudios}

http://www.garraioak.ejgv.euskadi.eus/r4130/es/ contenidos/evento/urbanismo inclusivo/es urbincl/ adjuntos/seminario es.pdf

Gobierno Vasco. Hiria Kolektiboa (2010): Manual de análisis urbano. Género y vida cotidiana. Manual metodológico para la realización de mapas de análisis urbanos desde la perspectiva de género y vida cotidiana de la ciudadanía. Mapas de la Ciudad Prohibida en municipios de la CAPV.

http://www.juntadeandalucia.es/economiayhacienda/planif_presup/genero/documentacion/Analisis urbano.pdf

INSTITUTO DE LA MUJER: Bibliografía de referencia para el análisis del urbanismo con perspectiva de género http://www.inmujer.gob.es/areasTematicas/redPoliticas/actividadesRed/docs/SesionPlenariaAviles/10 4 BibliogUrbanismoGenero.pdf

Muxi Martínez, Zaida (2015): "Mujeres haciendo ciudades: aprendiendo del pasado". Kultur: Revista
Interdisciplinària sobre cultura de la ciutat. Vol. 2, Núm. 3 (2015)

http://www.e-revistes.uji.es/index.php/kult-ur/ article/view/1720/1535

NNUU Nueva Agenda Urbana. Naciones Unidas, (2017):

http://habitat3.org/wp-content/uploads/ NUA-Spanish.pdf

SÁNChez de Madariaga, Inés (2004): Urbanismo con perspectiva de género. Fondo Social Europeo, Junta de Andalucía, 2004:

https://www.juntadeandalucia.es/institutodelamujer/ugen/sites/default/files/documentos/98.pdf

VVAA Género y Política Urbana (2017): Arquitectura y urbanismo desde la perspectiva de género [Libros]. Valencia: Instituto Valenciano de la Edificación.

http://www.five.es/descargas/archivos/urbanismo/ genero_y_politica_urbana_2017.pdf 